\title{
Critical finger ischemia and myocardial fibrosis development after sudden interruption of sildenafil treatment in a systemic sclerosis patient
}

\author{
C. Bruni ${ }^{1}$, S. Bellando-Randone', L. Gargani2 ${ }^{2}$, E. Picano ${ }^{2}$, \\ A. Pingitore ${ }^{2}$, M. Matucci-Cerinic' 1 , S. Guiducci ${ }^{1}$ \\ ${ }^{1}$ Department of Experimental and Clinical Medicine, Division of Rheumatology, University of Firenze, Italy; \\ 2Institute of Clinical Physiology, National Council of Research, Pisa, Italy
}

\begin{abstract}
SUMMARY
Systemic sclerosis (SSc) is a connective tissue disease frequently associated with Raynaud's Phenomenon (RP). Among possible pharmacological treatments, phosphodiesterase 5 inhibitors are considered in cases of severe non -responsive RP. We present the case of a male SSc patient wh presented with critical finger ischemia and concomitant appearance of myocardial fibrosis after sudden interruption of sildenafil treatment.
\end{abstract}

Key words: Systemic sclerosis; critical ischemia; phosphodiesterase 5 inhibitors; cardiac magnetic resonance imaging; myocardial fibrosis.

Reumatismo, 2016; 68 (2): 109-111

A sixty-year-old Caucasian man first presented to our center in December 2010 complaining of a 25 years history of Raynaud's phenomenon (RP), skin thickening of fingers and face, esophageal reflux and history of digital ulcerations; he was on atorvastatin and allopurinol for hypercholesterolemia and hyperuricemia, respectively.

On examination he presented calcinosis on the left elbow, sclerodactily with modified Rodnan skin score of 4/51 and facial telangiectasia. The positivity of anti-nuclear and anti-centromere antibodies and a late scleroderma pattern on nail fold videocapillaroscopy (1) supported the diagnosis and classification of systemic sclerosis (2). Internal organ baseline assessments excluded pulmonary involvement on chest high resolution computed tomography and lung function tests, while morphologic and functional cardiac abnormalities were ruled out on echocardiography and cardiac magnetic resonance imaging (MRI). The latter showed also absence of delayed contrast medium enhancement. Due to previous intolerance to calcium-channel blockers and refusal to intravenous iloprost, he was started on sildenafil $20 \mathrm{mg}$ three times a day for noncontrolled RP.

The treatment was beneficial until April 2011, when he stopped all oral medications for an episode of viral gastroenteritis; two days after he presented to emergency room with a critical ischemia of the right index finger: arterial doppler ultrasound showed completely absent blood flow in the second and third phalanx of the finger.

He was admitted to our unit and started on continuous intravenous iloprost and low molecular weight heparin, with rapid progressive improvement of the ischemic problem. A further arterial Doppler ultrasound showed a recovery of blood flow and repeated cardiac MRI showed delayed gadolinium enhancement at the inferior septum-ventricular junction (Figure 1). This suggested the presence of myocardial fibrosis with non-coronary distribution (patchy fibrosis) and without inducible
Corresponding author Bruni Cosimo Department of Experimental and Clinical Medicine Division of Rheumatology, University of Firenze Viale G. Pieraccini, 18 50139 Firenze, Italy E-mail: cosimobruni85@gmail.com 


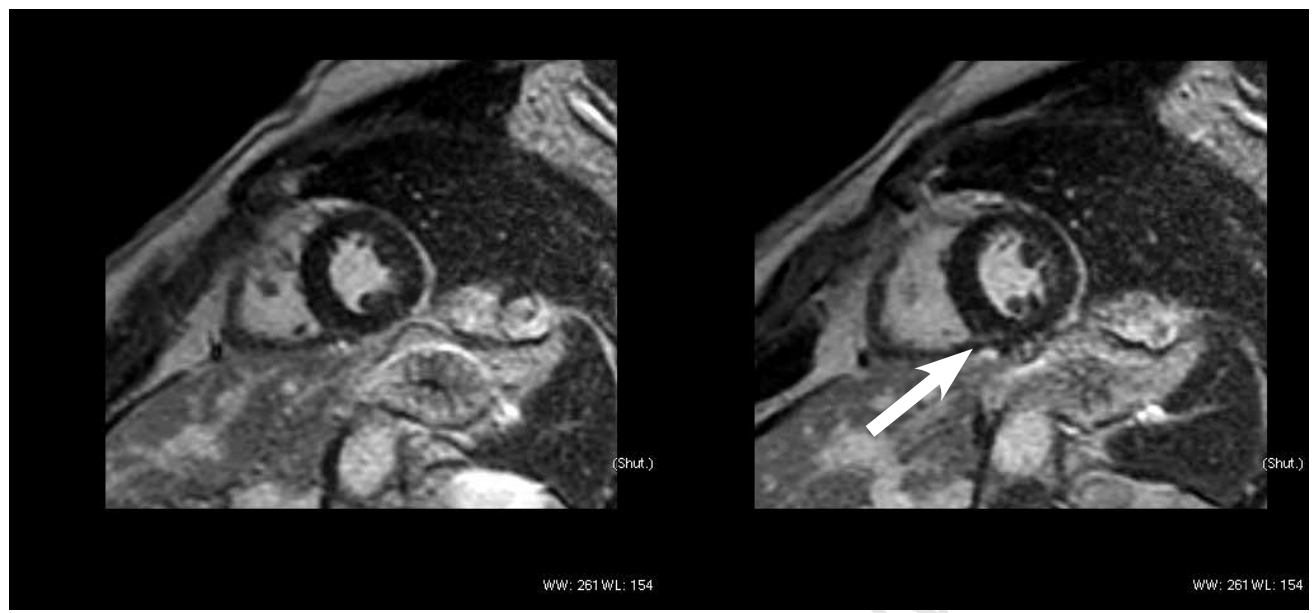

Figure 1 - Baseline (left) and follow up (right) cardiac magnetic resonance imaging with gadolinium enhancement studies images, the latter showing the appearence of delayed gadolinium enhancement (white arrow).

heart ischemia on perfusional miocardial single positron emission tomography later performed (3).

The patient was discharged with all previous medications plus prescription for monthly iloprost infusion and daily low dose aspirin.

To our knowledge, this is the first report of acute peripheral ischemic attack after sudden interruption of phosphodiesterase-5 inhibitors chronic vasodilating treatment (4), which led not only to ischemic finger suffering but also to tissue myocardial modifications $(5,6)$. In our case, cardiac MRI was useful in detecting early myocardial involvement.

MRI was previously suggested as a screening tool to analyze non-symptomatic scleroderma patients and identify subclinical involvement (7-9). According to the previously proposed hypothesis of myocardial $R P(10)$, we believe that the development of myocardial fibrosis could have been the effect of a sufferance of epicardial coronaries due to a prolonged vasospasm (11). The possibility of these two events being caused by a sildenafil withdraw rebound effect was never reported in previous clinical trials and therefore remains unproven.

Conflict of interest: the authors declare no potential conflict of interest.

\section{REFERENCES}

1. Cutolo M, Sulli A, Pizzorni C, et al. Nailfold videocapillaroscopy assessment of microvascular damage in systemic sclerosis. J Rheumatol. 2000; 27: 155-60.

2. van den Hoogen F, Khanna D, Fransen J, et al. 2013 classification criteria for systemic sclerosis: an American College of Rheumatology/European League against Rheumatism collaborative initiative. Arthritis Rheum. 2013; 65: 2737-47.

3. Nakajima K, Kawano M, Hasegawa M, et al. Myocardial damages in systemic sclerosis detected by gated myocardial perfusion SPECT and sympathetic imaging. Circ J. 2006; 70: 1481-7.

4. Roustit M, Blaise S, Allanore Y, et al. Phosphodiesterase-5 inhibitors for the treatment of secondary Raynaud's phenomenon: systematic review and meta-analysis of randomised trials. Ann Rheum Dis. 2013; 72: 1696-9.

5. Kahan A, Allanore Y. Primary myocardial involvement in systemic sclerosis. Rheumatology (Oxford). 2006; 45: iv14-7.

6. Allanore Y, Meune C. Primary myocardial involvement in systemic sclerosis: evidence for a microvascular origin. Clin Exp Rheumatol. 2010; 28: S48-53.

7. Ntusi NA, Piechnik SK, Francis JM, et al. Subclinical myocardial inflammation and diffuse fibrosis are common in systemic sclerosis - a clinical study using myocardial T1-mapping and extracellular volume quantification. J Cardiovasc Magn Reson. 2014; 16: 21.

8. Pingitore A, Guiducci S, Conforti ML, et al. Early detection of myocardial and pulmonary 
oedema with MRI in an asymptomatic systemic sclerosis patient: successful recovery with pulse steroid. Rheumatology (Oxford). 2013; 52: 1920-1.

9. Mavrogeni SI, Kitas GD, Dimitroulas T, et al. Cardiovascular magnetic resonance in rheumatology: current status and recommendations for use. Int J Cardiol. 2016; 217: 135-48. 10. Bulkley BH, Ridolfi RL, Salyer WR, et al.
Myocardial lesions of progressive systemic sclerosis. A cause of cardiac dysfunction. Circulation. 1976; 53: 483-90.

11. Mizuno R, Fujimoto S, Saito Y, Nakamura S. Cardiac Raynaud's phenomenon induced by cold provocation as a predictor of long-term left ventricular dysfunction and remodelling in systemic sclerosis: 7 year follow-up study. Eur J Heart Fail. 2010; 12: 268-75. 